\title{
Mathematics Anxiety and Math Instructional Time in First-Year Elementary School Teachers
}

\author{
Heidi L. Morton ${ }^{1}$ \\ University of Puget Sound \\ Cass Dykeman \\ Oregon State University
}

A Preprint

\begin{abstract}
Math anxiety is a pervasive problem that negatively impacts both children and adults. Teachers with math anxiety can unintentionally pass this anxiety on to their students; in addition, past studies have shown that these teachers may also end up spending less time engaged in math instruction. Both of these results can negatively impact their students for years to come. The present study examines the impact of a brief expressive writing intervention on three first-year elementary school teachers with math anxiety. Expressive writing interventions have been shown to have positive impacts on variables ranging from test anxiety to math performance. This multiple-baseline, multiple-probe, single-case research design examined the impacts of three 10-min expressive writing interventions on two variables: levels of math anxiety (as measured by the FS-ANX subscale of the Fennema-Sherman Mathematics Anxiety Scale) and the number of minutes engaged in math instruction. Results for both variables were mixed. Implications and recommendations for future research are discussed.
\end{abstract}

Keywords: math anxiety, expressive writing intervention, instructional time, first-year teachers, elementary school, math instruction

1. Correspondence concerning this article should be sent to Heidi Morton at hmorton@pugetsound.edu 


\section{Mathematics Anxiety and Math Instructional Time in First-Year Teachers}

Math anxiety can crush career aspirations and impede many other life opportunities. People who experience math anxiety tend to avoid advanced math classes and math-related careers. This avoidance limits their professional and personal opportunities and also deprives our nation and the world of people who might otherwise have contributed solutions to STEMrelated social, medical, and economic problems. A large percentage of mathanxious students first experience math anxiety in the early elementary grades. Many schoolteachers themselves are math-anxious and may end up passing math anxiety on to their students. Interestingly, elementary schoolteachers have higher math anxiety rates than other teachers.

Results of a previous study (Trice \& Ogden, 1986) showed a reduction in math instructional time provided by teachers with higher levels of math anxiety. This reduction in instructional time can negatively impact student learning. It has been 30 years since the Trice and Ogden study was first published. In that time, much has changed in the field of math pedagogy as well as our understanding of math anxiety. In addition, a number of brief interventions have been shown to hold promise for reducing math anxiety in adults. It is unknown if teacher math anxiety can be reduced by using a brief expressive writing intervention; it is also unknown if this brief intervention might have an impact on the time these teachers spend delivering math instruction in the classroom. Therefore, the purpose of this study is twofold: (a) to explore the impact of a brief writing intervention on the levels of math anxiety in first-year elementary school teachers and (b) to examine the potential impacts of the intervention on math instructional time delivered by first-year elementary school teachers.

A review of the literature on teacher math anxiety and teacher behavior revealed five key points that relate to this study: (a) elementary school teachers and preservice teachers have high levels of math anxiety, (b) math anxiety negatively impacts career and personal outcomes, (c) math anxiety has been shown to develop in early elementary years, (d) teacher time-on-task influences student outcomes, and (e) expressive writing interventions show promise for reducing the impacts of math anxiety. After 
the literature on the aforementioned points is reviewed, the research question for the current study will be presented.

Richardson and Suinn (1972) have described math anxiety as "feelings of tension and anxiety that interfere with the manipulation of numbers and the solving of mathematical problems in a wide variety of ordinary life and academic situations" (p. 551). Math anxiety is extremely pervasive; some estimates indicate that it may impact the majority of the general population and upward of $85 \%$ of future teachers (Finlayson, 2014; Jackson \& Leffingwell, 1999; Perry, 2004). Interestingly, elementary school teachers have particularly high levels of math anxiety (Battista, 1986; Bush, 1989). Kelly and Tomhave (1985) reported that female elementary education majors' anxiety levels were the highest compared to four other math-anxious college groups. This anxiety has serious negative consequences for teachers and students.

Math anxiety negatively impacts career and personal outcomes and has been linked with lower math achievement (Ashcraft \& Moore, 2009; Hembree, 1990; Ma, 1999). Many students avoid math-related careers or nonrequired math classes in high school due to the belief that they are just "bad at math" (Metje, Frank, \& Croft, 2007; Shapka et al., 2006). Math anxiety can persist 20 years or more (Jackson \& Leffingwell, 1999), continuing to impact major life decisions related to additional education and career choices, as well as daily life (Richardson \& Suinn, 1972; Shapka et al., 2006; Suri, Monroe, \& Koc, 2013). According to Ashcraft (2002), mathanxious people "tend to avoid college majors and career paths that depend heavily on math or quantitative skills, with obvious and unfortunate consequences" (p. 182). This math avoidance is not only personally tragic for those who may unnecessarily limit their career choices; it also hinders social and scientific advances through the loss of knowledge and human capital (Lyons \& Beilock, 2012). Hembree (1990) noted that "when otherwise capable students avoid the study of mathematics, their options regarding careers are reduced, eroding the country's resource base in science and technology." Due to these significant adverse outcomes, it is crucial to examine and address factors that may cause or increase math anxiety in students. For many students, this journey begins in the early years of their schooling. 
Math anxiety has been shown to develop in early elementary years. Ramirez, Gunderson, Levine, and Beilock (2013) found that in children as early as first and second grade, math anxiety is already associated with reduced math achievement. Researchers Jackson and Leffingwell (1999) explored the types of instructor behaviors that caused math anxiety in students ranging in grades from kindergarten through college. Participants were 157 preservice elementary teachers. Within this sample group, $93 \%$ ( $n$ $=146$ ) reported problems that resulted in the development of math anxiety. Of those who did report problems, $16 \%$ had their first traumatic encounter as early as Grades 3 and 4. A phenomenological study that same year focused on five highly math-anxious elementary preservice teachers; four of the five participants traced the roots of their struggles with math anxiety back to their own elementary school experiences (Hadfield \& Trujillo, 1999). These examples underscore the importance of examining factors during the early years of formal education, which could be causing or contributing to the development of math anxiety. One of those factors is the amount of time elementary school teachers spend teaching math.

Teacher time-on-task influences student learning outcomes. Many research studies have found that spending more time on instruction improves learning (Brown \& Saks, 1986; Silva, 2007; Young, 1980). One oftcited study examined 40 first-year elementary school teachers and found that teachers with higher levels of math anxiety spent up to $30 \%$ less time providing math instruction than those with lower or no math anxiety (Trice \& Ogden, 1986). These same teachers were 10 to 15 lessons behind their peers with their curriculum delivery. In a different study, Young (1980) found "there was a significant correlation $(p<.05)$ between the percentage of teacher time spent on academic tasks and students' reading achievement gains. Of all classroom process variables, time on task was the only one significantly affecting student performance." Another study found that both reading and mathematics test scores are significantly related to allocated instructional time (Brown \& Saks, 1986). Lavy's (2010) study found the following:

The effect of instructional time can be considered moderate or even large relative to other school level interventions for which we have reliable evidence. In the OECD sample, one additional hour of instruction increases on average test scores by about 0.15 of the within pupil standard deviation in test scores and by about 0.07 
standard deviation of the between pupil standard deviation (p. 2324).

Because of the consequences of instructional time on achievement and the demonstrated impact of math anxiety on math instructional time provided to students, it is important to examine potential interventions that could either reduce teacher math anxiety or mitigate the impacts of teacher math anxiety on math instructional time, or both.

Expressive writing interventions show promise for reducing the impacts of math anxiety. Sometimes referred to as written disclosure (WD) or written emotional disclosure (WED), expressive writing has been found to be an effective intervention for coping with many conditions and experiences, including trauma and loss (Bragdon \& Lombardo, 2012; Pennebaker, 2000; Pennebaker \& Beall, 1986; Sloan, Marx, \& Greenberg, 2011). Some studies have revealed increased health benefits such as improved management of chronic pain, asthma, and arthritis (Pennebaker, 2000; Smyth, Stone, Hurewitz, \& Kaell, 1999) and improved immune functioning (Frattaroli, 2006; Pennebaker, 1989). Expressive writing interventions are said to be similar to traditional exposure therapy as far as the mechanism of therapeutic impact (Sloan et al., 2011).

A recent study was conducted to examine whether an expressive writing activity would improve the academic performance of high school students with test anxiety. The premise was that an expressive writing activity would "help to alleviate the intrusive thoughts that test-anxious students experience and thus would free up their working memory resources for the upcoming test" (Maloney, Sattizahn, \& Beilock, 2014, p. 408). Those who participated in the expressive writing intervention had higher overall scores than those who did not. Also of interest was that the students who were most text-anxious were the ones who benefitted the most from the intervention. Another study found that it was expressive writing in particular (versus writing about a prompt unrelated to the thoughts and feelings of the upcoming test) that accounted for the improved exam scores (Ramirez \& Beilock, 2011). Although test anxiety is a separate construct from math anxiety, these two forms of anxiety share many similarities, including their impacts on working memory and resulting performance tasks (Mavilidi, Hoogerheide, \& Paas, 2014; Morsanyi, Busdraghi, \& Primi, 2014; Suárez-Pellicioni, Núñez-Peña, \& Colomé, 2015). 
Another study focused on the use of expressive writing as an intervention specifically for students with math anxiety (Park, Ramirez, \& Beilock, 2014). Results indicated a significant reduction in math anxiety and improved math performance after a single 7-min session of expressive writing directly preceding a test. These studies indicate that an expressive writing intervention can be a successful, efficient, and cost-effective way to mitigate the impacts of math anxiety and increase math performance.

Given the importance of a workforce and individuals with strong math and math-related skills, it is critical to examine ways to both reduce math anxiety and increase math instructional time. Therefore, the purpose of this study is to address two research questions: (a) What is the impact of a brief expressive writing intervention on first-year elementary school teachers with math anxiety? (b) What is the impact of a brief expressive writing intervention on math instructional time provided by first-year elementary school teachers with math anxiety?

\section{Method}

\section{Design}

To address the research questions guiding this study, the researchers used a single-subject (also known as single-case), multiple baseline, multiple probe design (Christ, 2007; Heppner, Wampold, \& Kivlighan, 2008; Horner \& Baer, 1978). The principal researcher used an online random number generator (https://www.random.org/) to assign baseline lengths of 3, 5, and 7 days respectively for Participants A, B, and C. Baseline stability was determined using criteria by Gast and Spriggs (2010) and Perone (1991). The stability envelope criteria used was that $80 \%$ of the data points needed to fall within $20 \%$ of the median score within the phase for the baseline to be considered stable. If baseline stability was not reached by the assigned amount of baseline data points, baseline measurements continued until either baseline stability as defined by Gast and Spriggs (2010) was evident or Perone's (1991) fixed time-interval stability criteria were met. The independent variable was an expressive writing intervention following a protocol similar to that used by Pennebaker (1989). The dependent variables were (a) math instructional time provided to students by the teacher and (b) teacher math anxiety. 


\section{Participants}

The participants were three first-year teachers in Grades 3 and 5 within three different elementary schools in a large, suburban school district in on the West Coast. Participants were recruited via an e-mail sent to all first-year second-to-fifth-grade elementary school teachers throughout the district, as identified by district officials. Selection of participants was based on the following criteria: the first three interested first-year elementary teachers in Grades 2 to 5 who had a score of 22.9 or above on the screening instrument (Abbreviated Mathematics Anxiety Scale) and consented to participate in the study.

Participant A. Participant A was a 25-year-old Caucasian female. Her AMAS score was 25, indicating a moderate level of math anxiety. She shared that she had struggled with math from elementary school through college, having difficulties understanding the concepts and feeling like there was little support - either cognitive or affective - to help her become more proficient and comfortable with math.

Participant B. Participant B was a 23-year-old Caucasian female. She shared that her first negative experience with math was in the second grade. She has developed more confidence with basic math as an adult, though she still experiences a lot of anxiety about both completing math problems and teaching math. Her initial AMAS score was a 24, the lowest of the three participants.

Participant C. Participant C was a 50-year-old Caucasian female. She reported that while she had never really enjoyed math, her difficulties in understanding math began during sixth grade and with fractions, and she remembered encountering a lot of difficulty with math classes in high school. Her initial AMAS score was a 27, indicating a high-moderate level of math anxiety.

\section{Measures}

Abbreviated Mathematics Anxiety Scale (AMAS). To screen potential participants for qualifying levels of math anxiety, the AMAS was 
administered to respondents interested in participating in the study. The AMAS has only nine items and requires students to rate their responses using a fully anchored 5-point Likert scale, ranging from 1 (low anxiety) to 5 (high anxiety). The total score represents the level of math anxiety; high scores indicate high levels of math anxiety. Isiksal, Curran, Koc, and Askun (2009) found in a large sample of U.S. preservice elementary school teachers a mean of $22.9(S D=5.7)$. Total scores were calculated for each participant, and the results are shared in the Findings and Conclusion sections. Respondents with an AMAS score at or above 22.9 (based on cutoff scores as classified by Morsanyi et al., 2014) were considered to have met math-anxiety criteria for inclusion in the study. The AMAS shows excellent internal consistency (Cronbach's $\alpha=.90$ ) and test-retest reliability ( $r=.85$ over a 2 -week period); there is also strong evidence of convergent validity through correlation with a well-established math anxiety measure, the Math Anxiety Rating Scale - Revised $(r=.85)$ (Hopko, Mahadevan, Bare, \& Hunt, 2003). Due to its psychometric soundness and its convenient nine-item scale, "the AMAS appears to be the test of choice for future work on math anxiety" (Ashcraft \& Ridley, 2005, as cited in Eden, Heine, \& Jacobs, 2013).

Fennema-Sherman Mathematics Anxiety Scale-Revised: anxiety subscale (FS-ANX). Participants completed the anxiety subscale (FS-ANX) of the FMAS-R to measure levels of math anxiety throughout the baseline and intervention phases. The FSMAS-R is a nine-item, two-factor scale designed to measure math anxiety in classroom-based settings (Lim \& Chapman, 2013; Ren, Green, \& Smith, 2016). The FSMAS-R has two subscales: EASE (four items related to feelings of ease with mathematics) and ANX (five items related to anxiety with mathematics). Psychometric properties of the FSMAS-R have been shown to be favorable, with the subscales displaying good internal consistencies and satisfactory test-retest reliability over a 1-month period. Correlations between the subscales and between theoretically related constructs support the construct validity of the FSMAS-R (Lim \& Chapman, 2013). FS-ANX scores were calculated for each data point in both Phase $\mathrm{A}$ and $\mathrm{B}$ and are included in the results section.

\section{Apparatus}


Harvest. Participants tracked their time engaged in actual math instruction by using the Harvest time-tracking software application, which was made available to participants at no cost. As participants logged their time, the recorded logs were available immediately to the researchers. The Harvest application was chosen for its ease of use, its cross-platform availability, its reputation for stability, and its ability to encrypt, verify, and automatically store collected data.

Qualtrics. To complete the AMAS screening assessment, the FSANX assessments during baseline and intervention phases, and the expressive writing interventions, participants utilized the online survey platform Qualtrics (2016). For each item of both the AMAS and the FSANX, the participant selected the radio button option that best described his or her answer. For the expressive writing interventions, participants typed within a text box for the allotted 10-min period. The software program was configured to save their text answers and close the text box after 10 min of writing had elapsed.

\section{Intervention}

Throughout the baseline period, participants recorded their levels of math anxiety by completing the FS-ANX via Qualtrics and also tracked math instructional time using the Harvest application. Baseline stability was determined using both variables. Once the minimum assigned baseline days had been completed, baseline stability was assessed, and determinations were made as to when participants could move into the intervention phase.

The intervention consisted of three brief expressive writing sessions. Three times over the course of two weeks (typically on Mondays and Thursdays), participants navigated to the Qualtrics survey online and responded to the following writing prompt:

Please take the next 10 minutes to write as openly as possible about your deepest thoughts and feelings regarding math. Thinking about math - whether it is completing math problems, taking math classes, teaching math to students, or recalling past experiences in your own math classes - can evoke many different feelings and responses. The important thing is that you really let go and dig down to your very deepest emotions and thoughts and explore them in your writing. 
For the second and third interventions, the following statement was added as the second-to-last sentence in the prompt: "You can write about the same topic or event as you did the previous week, exploring it further, or you can write about a different topic or event." At the end of the $10 \mathrm{~min}$, the text box closed, and participants' responses were saved within Qualtrics.

Procedures. Participants were instructed to complete the FS-ANX online twice each week during both the baseline and intervention phases, as well as record their time spent giving math instruction that same day. These data collection days were scheduled to take place either on Mondays and Thursdays or Tuesdays and Fridays, depending on which option worked best for each participant. For the intervention phase, the expressive writing prompts were scheduled during the evenings before the designated datacollection days. E-mails and text-message reminders were sent to participants $30 \mathrm{~min}$ before the start of the writing interventions as well as the mornings of data-collection days.

Intervention fidelity. To ensure a greater degree of fidelity to the intervention and with data collection, several processes were employed. First, participants were given written instructions outlining the datacollection process and how to use Harvest and Qualtrics. Participants had the opportunity to practice tracking their math instructional time for a week before the start of the study; they were also offered individual sessions with the student researcher to clarify any questions they may have had about the study and the process. This process helped ensure that participants knew how to access and use the apparatus and that they could incorporate the use of the application within their work setting and work routines. Second, math instructional time was explicitly defined for participants as the number of minutes per day that the teacher was actively engaged with students in either direct whole-group math instruction (e.g., lecturing on a mathematical concept or lesson) or otherwise actively facilitating learning opportunities (checking for understanding or facilitating individual-, group-, or classroom-level mathematics-learning opportunities). Time students spent independently working on mathematical problems without direct and active teacher engagement (e.g., students completing math problems or a worksheet while the teacher was engaged in a different task) was not counted as math instructional time. Third, participants were instructed to complete the writing intervention within a 2-hr window in the 
evenings of the chosen intervention dates. Fourth, the intervention writing response time was limited to $10 \mathrm{~min}$ for all sessions and participants. Fifth, participants were reminded to complete the writing intervention with fidelity and with minimal distractions.

\section{Data Analysis}

Data analysis was conducted through both visual analysis and nonoverlap of all pairs (NAP). Lane and Gast's (2014) protocol was utilized for visual analysis to examine levels, trends, and stability within and across conditions. NAP analysis was conducted through the use of an online NAP calculator (Vannest, Parker, \& Gonen, 2011). The alpha level for all NAP analyses was set at .05.

\section{Results}

Participants completed the study in the spring of 2017. Graphical representation of the results can be found in Figures 1 and 2 .

\section{Math Anxiety}

Visual analysis of the data for Participant A reflected no changes in level of math anxiety both within and between phases, $(\bar{x}=20, S D=0$, for both phases). All data points in the baseline and intervention phases fell within the calculated stability envelope. NAP analysis resulted in a NAP calculation of 0.50 , with a $p$-value of 1.0 .

For Participant B, visual analysis indicated a slight decrease in the FS-ANX scores from baseline during the intervention phase $(\bar{x}=16.00, S D=0$ in intervention phase vs. $\bar{x}=16.83, S D=0.75$ in baseline phase). Betweencondition analysis shows a change from a decreasing trend in the baseline phase to a flat, stable trend in the intervention phase. The NAP calculation was 0.17 , with a $p$-value of 0.12 .

Data for Participant $\mathrm{C}$ showed a trend of decreasing levels of math anxiety over the three points in the intervention phase. However, the mean level of math anxiety in the intervention phase $(\bar{x}=15.0, S D=1.0)$ was higher 
than that in the baseline phase $(\bar{x}=14.6, S D=1.52)$. The calculated NAP score for FS-ANX data was 0.57, with a $p$-value of 0.77 .

\section{Math Instructional Time}

The trend lines for Participant A shows an increase in math instructional time during the baseline phase $(\bar{x}=55.50 \mathrm{~min}, S D=11.50)$ and a decrease during the intervention phase $(\bar{x}=26.3 \mathrm{~min}, S D=5.51)$. NAP analysis calculation was 0.00 , with a $p$-value of 0.02 .

For Participant B, data for teacher time engaged in math instruction showed a decrease in time spent in math instruction during the intervention phase $(\bar{x}=48.33, S D=10.41)$ as compared to the baseline phase $(\bar{x}=60$, $S D=16.73)$. The NAP calculation was 0.28 , with a $p$-value of 0.30 .

Data for Participant $\mathrm{C}$ showed an increase in teacher instructional time during the intervention phase $(\bar{x}=37.67, S D=6.81)$ as compared to the baseline phase $(\bar{x}=35.60, S D=5.37)$. NAP analysis resulted in a NAP calculation of 0.53 , with a $p$-value of 0.88 .

\section{Discussion}

The purpose of this study was to answer two questions: (a) What is the impact of a brief expressive writing intervention on first-year elementary school teachers with math anxiety? (b) What is the impact of a brief expressive writing intervention on math instructional time provided by firstyear elementary school teachers with math anxiety?

\section{Math Anxiety}

Overall, between-phase scores on the FS-ANX reflected mixed results, ranging from no change to slight reductions in math anxiety. Within-phase analysis during the intervention phase indicated no changes for two participants and an improving, decreasing trend for the third participant. Intervention phase level data for the third participant showed an initial increase at the start of the intervention phase, with decreasing levels in the remainder of the phase. 
Participant A's FS-ANX scores reflected no variability across time. This data may potentially indicate a response bias or even a type of ceiling effect, given that her FS-ANX scores were the highest of the three participants. Each of the 13 combined scores in the study was a score of 20 . Even when examining differences within individual item scores, there are only two exceptions, both of which occurred during the first week. During this week, the participant marked a 5 (Strongly Agree) on one item and a 3 (Neither Agree nor Disagree) on another. At all other times throughout the study, every item-level response was a 4 on the 5-point scale. However, the mean survey-completion time throughout the study (33 s) was quite similar for all three participants when obvious outliers were excluded, which may not support the hypothesis of response bias for Participant A.

Participant B's levels of math anxiety showed a declining trend within the baseline phase. Because anxiety levels were already decreasing before the intervention began, any reduction in anxiety levels postintervention cannot safely be attributed solely to the intervention. There may be the possibility of subject effects here. In particular, the participant may be responding to the test conditions, indicating a potential Hawthorne effect (Vogt, Johnson, \& Burke, 2016).

Participant $\mathrm{C}$ was the only participant whose scores reflected changes in both variables in the expected directions. However, in general, the levels of math anxiety were higher than during the intervention phase, which was not expected. It is possible that the levels may continue to drop postintervention as more time passes, as discussed in following sections.

\section{Math Instructional Time}

The results for the math instructional time variable were unexpected. All three participants' data varied widely throughout both phases of the study. Trend lines for Participants A and B showed increasing time in the baseline phase and decreasing time spent during the intervention phase, while trend lines for Participant B showed the opposite. The researchers have some hypotheses as to why the data may have been so variable; these hypotheses are discussed further in the Limitations section. 
A question to consider is whether the expressive writing interventions were too short in duration or number to make a noticeable change in levels of math anxiety. While Pennebaker's earlier studies included expressive writing interventions that were 15-20 min each in duration over three consecutive days (Pennebaker, 1989, 1990), more recent studies have seen positive results in reducing math and test anxiety after a single expressive writing session as short as 5-10 min (Doherty \& Wenderoth, 2017; Park et al., 2014; Ramirez \& Beilock, 2011). It is possible that the number or length (dosage) of the intervention sessions was not sufficient to produce change. While some conditions have changed after a single brief intervention session (Greenberg et al., 1996, as cited in Smyth \& Pennebaker, 2008), the nature and persistence of anxiety-related conditions (American Psychiatric Association, 2013) might require more expressive writing time or sessions than were used in the present study.

Multiple scheduling conflicts were experienced by the participants throughout the study, resulting in changes to their initially planned data collection days. Most of these related to changes in their teaching schedules because of unanticipated conflicting tasks, trainings, and demands related to the end of the school year, including the several-week-long mandatory state testing window. The authors hypothesize that the decrease in math instructional time during the intervention phase for Participants A and B may reflect the time of year the study was conducted rather than reflecting the impacts of the expressive writing intervention.

\section{Limitations}

There are several limitations to the study. First, the study was conducted with only three participants, often considered the minimum acceptable number for single-case research to demonstrate experimental control (Ray, 2015; Horner et al., 2005). In addition, the participants were not selected randomly but were instead based on volunteers in a specific geographical area who expressed interest in participating. The lack of random selection increases the likelihood that the sample might be biased. For example, participants may have been motivated by the desire to help specific individuals, such as the researcher or one or more of their current students struggling in math. The sample could have also inadvertently reflected individual differences within local teacher education programs, or 
any number of differences that are not controlled for when using a convenience sample. Second, there were numerous challenges related to the field research setting that may have introduced extraneous variables or otherwise impacted fidelity to the initially designed protocol. These challenges are discussed in the following pages. Third, the study relied on self-reported data for teacher math instructional time. With self-reported data, there is always the risk that the data could be biased due to factors such as selective memory (for those times when participants failed to use the Harvest timer and instead relied on their recollections at the end of the collection day) as well as potential response bias. Fourth, it is unknown whether the participants adhered to the definition of math instructional time throughout the study when recording their time. Fifth, the data for math instructional time was based on single days during the workweek. Sixth, it is possible that the time between the series of interventions and the measured variables was insufficient to demonstrate potential change. Seventh, it is unknown whether completing the intervention the evening prior to measuring math anxiety and instructional time could have impacted the results. Several previous expressive writing studies have administered the intervention immediately prior to a performance task. Eighth, it is strongly suspected that the timing of the study impacted results and the ability to measure the effects of the intervention accurately. The end of the school year is a challenging and hectic time, complicating the ability to control for unintended influences on the study. These influences may have impacted both the adherence to the protocol and the accuracy of the data collected. Some of these limitations are discussed in greater detail below.

Procedural fidelity. Despite attempts to control for confounding variables, there were differences between the planned and actual datacollection processes during the study. Many of these differences can be attributed to the vicissitudes of field research (as compared to research in more controlled settings, such as a lab). The impact of these differences on the results of the study is unknown; however, some of the differences may have increased threats to validity and reliability. The differences included unanticipated teacher/staff-training days, required state-testing timelines, reversed order of data collection, and changes in the time between the expressive writing intervention and the FS-ANX completion. The study took place during a particularly challenging time of year, as teachers were 
approaching the end of the school year and facing numerous schedule changes and deadlines.

Adhering to the planned timing of the interventions and datacollection days appeared to be problematic for all three participants within their field settings. All three participants requested and received both e-mail and text-message reminders at the designated writing-intervention times and data-collection times. Despite receiving these reminders, Participants A and $\mathrm{C}$ diverged from the planned timing for completing the intervention for all three sessions. The original intent was for participants to complete the intervention the evening before measuring math anxiety and instructional time, to allow for some separation. However, Participants $\mathrm{A}$ and $\mathrm{C}$ completed the interventions the day after they received the reminders (in other words, on the same days they reported FS-ANX and time data), with the mean time between completing the intervention and taking the FS-ANX being less than 7 min. Only Participant B completed the interventions the evening before data collection as planned, and that was true only of the first and third interventions; for the second intervention, Participant B reversed the order and recorded FS-ANX and time data the day before she completed the intervention. The reasons for these divergences are unknown. It is suspected that at least on some of these occasions, participants may have forgotten until the data-collection reminders the next day. It is unknown if following the suggested protocol would have impacted the resulting FS-ANX scores and instructional times recorded.

Participant B deviated from the planned twice-per-week datacollection schedule, sometimes taking the FS-ANX up to four times in a single week. To try and match the rates of collection with the other two participants and with the original protocol, only data from the two originally chosen data-collection days per week were included in the data analysis. For this reason, the results for Participant B are more vulnerable to threats to reliability such as memory effect. Though the Perone (1991) protocol for progressing from the baseline phase would have allowed participants to begin the intervention at an earlier point in time than occurred during the study, additional baseline points were collected in the hopes that a stable baseline for both dependent variables could be established before beginning the intervention phase. Baseline stability for math instructional time was difficult to establish for Participants A and C 
due to the widely varying data reported for time engaged in math instruction. After additional baseline points were collected for Participants A and B and stability (as defined using Gast and Spriggs' 2010 protocol) still did not emerge for the math instructional time variable, the researchers decided to proceed with the intervention phase per the Perone (1991) protocol.

There were two occasions when participants did not track their math instructional time live using the Harvest timer and instead calculated a time estimate at the end of their day. It is unknown whether these time figures were as accurate as those tracked using the Harvest timer.

Also unknown is the actual conditions under which the participants completed the expressive writing interventions. In previously mentioned studies based on expressive writing (Pennebaker, 1989; Pennebaker \& Beall, 1986; Pennebaker et al., 1990), the conditions during the intervention were carefully controlled in settings with conditions more similar to those in a laboratory. While the current study instructed participants to complete the intervention during a time in the evening, outside their regular workday, and with minimal distractions, it is unknown under what conditions the participants actually completed the intervention.

Participants. Participants were selected for inclusion in the study based on their initial AMAS scores, with 22.9 being the minimum qualifying score. Participant scores for the current study were 25, 24, and 27. Given that the range of possible scores for the AMAS is 0 to 45 , it is possible that recruitment of participants with higher scores and thus higher levels of math anxiety may have resulted in more noticeable differences between baseline and intervention stages.

The sample population consisted of three Caucasian women in a single suburban geographical area within the state of Washington. It is unknown whether sample populations with different demographics, geographic locations, and school settings would have impacted the results.

\section{Implications for Future Research}


The researchers offer several suggestions should a similar study be conducted in the future. While there are inevitable complications when conducting field research, examining additional ways to reduce the likelihood of these confounding factors would be strongly recommended for replication studies in the future.

One factor that introduced a number of confounding variables was the time of year during which the study was conducted. There were numerous daily and weekly changes to schedules and routines during the busy end of the school year. At the very least, avoiding the time of year when the mandatory annual state achievement tests are administered would eliminate many of these scheduling impacts in future studies.

A poststudy review of the fidelity to the research protocol would have helped examine whether participants adhered to the definition for the variable of math instructional time as outlined in the original instructions, potentially impacting the data reported. Also, a more representative figure for math instructional time may have resulted if the mean daily time over the course of several days or a full workweek was used for each data point as opposed to data collected from a single day at a time. Arranging a time to connect and check in with participants after the first or second day of data collection may have also resulted in more frequent adherence to the protocol and timing of data collection.

Math-instructional-time data was collected through the use of teacher self-reports. While commonly used in the social sciences, selfreported data can be fallible (Schwarz, 1999). Employing the use of observers to collect this data, or video or audio recording and analyzing classroom instructional time, could improve the reliability of the results.

One question for future research is whether more time may need to pass after the intervention is completed before measurable positive impacts occur. Unlike with typical mechanisms of catharsis, expressive writing prompts may not produce an immediate sense of improved affect. In a previous study by Pennebaker (1989), self-reports indicated increased feelings of sadness after completing expressive writing prompts, yet when queried 4 months later, participants indicated improvements in symptoms, that the studies had been valuable and meaningful to them, and 93\%-98\% 
indicated they would participate again (p. 218). In another study, Pennebaker found positive effects for those in the experimental (expressive writing intervention) group as compared to those in the control group as measured five or more months after the intervention (Pennebaker, Colder, \& Sharp, 1990). With the present study, of note in the results is that Participant $\mathrm{C}$ showed a steadily decreasing trend in levels of math anxiety during the intervention phase, yet overall level of math anxiety was higher during this phase than the baseline phase. These results might be similar to what Pennebaker found regarding increased initial symptoms with improvement demonstrated over a more extended period of time.

Participant B also showed a decreasing trend; it would be interesting to see where participants' levels of math anxiety were 4 months or more after the intervention. Still, some studies have shown immediate impacts directly following an expressive writing intervention. However, these studies focused on variables other than levels of math anxiety, such as math performance (Park et al., 2014; Ramirez \& Beilock, 2011), test anxiety (Doherty \& Wenderoth, 2017), and number of illness visits to a health clinic (Pennebaker, Colder, \& Sharp, 1990). More research is needed to determine if positive results may occur after a similar protocol used in the present study but after more time passes after the intervention concludes. For future studies, follow-up measurements of both math anxiety and math instructional time are recommended at 4- and 6-month intervals after the intervention period. This action would help examine the possibility of a delayed treatment effect as has sometimes been seen with other expressive writing intervention studies.

It is recommended that there be more than three participants in future studies to strengthen experimental control. Future studies might include samples from other genders as well as from other parts of the country. It would also be interesting to examine additional participant factors, such as the graduate program through which the teachers received their training, engagement in first-year teacher-mentor support programs, and the reported initial onset of math anxiety.

Within the sample population, there is the opportunity for history effects to play a role in the outcomes. First-year teachers experience a rapid learning curve and tremendous professional growth during the school year. 
Although the researchers do not suspect that history effects played a part in the results of the current study, the potential for history effects should be kept in mind when conducting replication studies or related studies with first-year teachers. 


\section{References}

Ashcraft, M. H. (2002). Math anxiety: Personal, educational, and cognitive consequences. Current Directions in Psychological Science, 11, 181185. Retrieved from http://www.jstor.org/stable/20182804

Ashcraft, M. H., \& Moore, A. M. (2009). Mathematics anxiety and the affective drop in performance. Journal of Psychoeducational Assessment, 27, 197-205. doi:10.1177/0734282908330580

Battista, M. T. (1986). The relationship of mathematics anxiety and mathematical knowledge to the learning of mathematical pedagogy by preservice elementary teachers. School Science and Mathematics, 86, 10-19. doi:10.1111/j.1949-8594.1986.tb11580.x

Bragdon, R. A., \& Lombardo, T. W. (2012). Written disclosure treatment for posttraumatic stress disorder in substance use disorder inpatients. Behavior Modification, 36, 875-896. https://doi.org/10.1177/0145445512451273

Brown, B. W., \& Saks, D. H. (1986). Measuring the effects of instructional time on student learning: Evidence from the beginning teacher evaluation study. American Journal of Education, 94, 480-500. Retrieved from http://www.jstor.org/stable/1085338

Bush, W. (1989). Mathematics anxiety in upper elementary school teachers. School Science and Mathematics, 89, 499-508.

Christ, T. J. (2007). Experimental control and threats to internal validity of concurrent and nonconcurrent multiple baseline designs. Psychology in the Schools, 44, 451-459. https://doi.org/10.1002/pits.20237

Doherty, J. H., \& Wenderoth, M. P. (2017). Implementing an expressive writing intervention for test anxiety in a large college course. Journal of Microbiology \& Biology Education, 18. Retrieved from https://www.ncbi.nlm.nih.gov/pmc/articles/PMC5576769/

Eden, C., Heine, A., \& Jacobs, A. M. (2013). Mathematics anxiety and its development in the course of formal schooling - a review. Psychology, 4, 27-35. doi:10.4236/psych.2013.46A2005

Finlayson, M. (2014). Addressing math anxiety in the classroom. Improving Schools, 17, 99-115. doi:10.1177/1365480214521457

Frattaroli, J. (2006). Experimental disclosure and its moderators: A metaanalysis. Psychological Bulletin, 132, 823-865. doi:10.1037/00332909.132.6.823 
Gast, D. L., \& Spriggs, A. D. (2010). Visual analysis of graphic data. In D. L. Gast (Ed.), Single subject research methodology in behavioral sciences (pp. 199-233). New York, NY: Routledge.

Hadfield, O. D., \& Trujillo, K. M. (1999). Tracing the roots of mathematics anxiety through in-depth interviews with preservice elementary teachers. College Student Journal, 33, 219.

Hembree, R. (1990). The nature, effects, and relief of mathematics anxiety. Journal for Research in Mathematics Education, 21, 33-46. http://doi.org/10.2307/749455

Heppner, P., Wampold, Bruce E., \& Kivlighan, D. M. (2008). Research design in counseling (3rd ed.). Belmont, CA: Thomson Brooks/Cole.

Hopko, D. R., Mahadevan, R., Bare, R. L., \& Hunt, M. K. (2003). The abbreviated math anxiety scale (AMAS) construction, validity, and reliability. Assessment, 10, 178-182. https://doi.org/10.1177/1073191103010002008

Horner, R. D., \& Baer, D. M. (1978). Multiple-probe technique: A variation of the multiple baseline. Journal of Applied Behavior Analysis, 11, 189-196. https://doi.org/10.1901/jaba.1978.11-189

Horner, R. H., Carr, E. G., Halle, J., McGee, G., Odom, S., \& Wolery, M. (2005). The use of single-subject research to identify evidence-based practice in special education. Exceptional Children, 71, 165-179. https://doi.org/10.1177/001440290507100203

Isiksal, M., Curran, J. M., Koc, Y., \& Askun, C. S. (2009). Mathematics anxiety and mathematical self-concept: Considerations in preparing elementary-school teachers. Social Behavior and Personality: An International Journal, 37, 631-643. doi:10.2224/sbp.2009.37.5.631

Jackson, C. D., \& Leffingwell, R. J. (1999). The role of instructors in creating math anxiety in students from kindergarten through college. Mathematics Teacher, 92, 583-586.

Kelly, W. P., \& Tomhave, W. K. (1985). A study of math anxiety/math avoidance in preservice elementary teachers. Arithmetic Teacher, 32, 51-53.

Lane, J. D., \& Gast, D. L. (2014). Visual analysis in single case experimental design studies: Brief review and guidelines. Neuropsychological Rehabilitation, 24, 445-463. https://doi.org/10.1080/09602011.2013.815636

Lavy, V. (2010). Do differences in school's instruction time explain international achievement gaps in maths, science and language? 
Evidence from developed and developing countries. Centre for the Economics of Education, LSE. Retrieved from http://econpapers.repec.org/paper/cepceedps/0118.htm

Lim, S. Y., \& Chapman, E. (2013). An investigation of the FennemaSherman Mathematics anxiety subscale. Measurement and Evaluation in Counseling and Development, 46, 26-37. https://doi.org/10.1177/0748175612459198

Lyons, I. M., \& Beilock, S. L. (2012). Mathematics anxiety: Separating the math from the anxiety. Cerebral Cortex, 22(9), 2102-2110. http://doi.org/10.1093/cercor/bhr289

Ma, X. (1999). A meta-analysis of the relationship between anxiety toward mathematics and achievement in mathematics. Journal for Research in Mathematics Education, 30, 520-540. doi:10.2307/749772

Maloney, E. A., Sattizahn, J. R., \& Beilock, S. L. (2014). Anxiety and cognition. Wiley Interdisciplinary Reviews: Cognitive Science, 5, 403411. https://doi.org/10.1002/wcs.1299

Mavilidi, M.-F., Hoogerheide, V., \& Pass, F. (2014). A quick and easy strategy to reduce test anxiety and enhance test performance. Applied Cognitive Psychology, 28, 720-726. https://doi.org/10.1002/acp.3058

Metje, N., Frank, H. L., \& Croft, P. (2007). Can't do maths-understanding students' maths anxiety. Teaching Mathematics and Its Applications, 26, 79-88. doi:10.1093/teamat/hrl023

Morsanyi, K., Busdraghi, C., \& Primi, C. (2014). Does maths anxiety make people bad decision-makers? The link between mathematical anxiety and cognitive reflection. In P. M. Bello, M. Guarini, M. McShane, \& B. Scassellati (Eds.), Proceedings of the 36th Annual Meeting of the Cognitive Science Society, 1042-1047. Austin, TX: Cognitive Science Society.

Park, D., Ramirez, G., \& Beilock, S. L. (2014). The role of expressive writing in math anxiety. Journal of Experimental Psychology: Applied, 20, 103-111. https://doi.org/10.1037/xap0000013

Pennebaker, J. W. (1989). Confession, inhibition, and disease. Advances in Experimental Social Psychology, 22, 211-244.

https://doi.org/10.1016/S0065-2601(08)60309-3

Pennebaker, J. W. (2000). Telling stories: The health benefits of narrative. Literature and Medicine, 19, 3-18. https://doi.org/10.1353/lm.2000.0011 
Pennebaker, J. W., \& Beall, S. K. (1986). Confronting a traumatic event: Toward an understanding of inhibition and disease. Journal of Abnormal Psychology, 95, 274-281. https://doi.org/10.1037/0021843X.95.3.274

Pennebaker, J. W., Colder, M., \& Sharp, L. K. (1990). Accelerating the coping process. Journal of Personality and Social Psychology, 58, 528-537. https://doi.org/10.1037/0022-3514.58.3.528

Perone, M. (1991). Experimental design in the analysis of free-operant behavior. In I. H. Iversen \& K. A. Lattal (Eds,), Experimental analysis of behavior, part 1 (pp. 135-171). New York, NY: Elsevier Science.

Perry, A. B. (2004). Decreasing math anxiety in college students. College Student Journal, 38, 321.

Qualtrics. (2016). Qualtrics [Computer software]. Provo, UT: Author.

Ramirez, G., \& Beilock, S. L. (2011). Writing about testing worries boosts exam performance in the classroom. Science, 331(6014), 211-213. https://doi.org/10.1126/science.1199427

Ramirez, G., Gunderson, E., Levine, S., \& Beilock, S. (2013). Math anxiety, working memory, and math achievement in early elementary school. Journal of Cognition and Development, 14, 187-202. doi:10.1080/15248372.2012.664593

Ray, D. C. (2015). Single-case research design and analysis: Counseling applications. Journal of Counseling \& Development, 93, 394-402. https://doi.org/10.1002/jcad.12037

Ren, L., Green, J. L., \& Smith, W. M. (2016). Using the Fennema-Sherman Mathematics Attitude Scales with lower-primary teachers. Mathematics Education Research Journal, 28(2), 303-326. https://doi.org/10.1007/s13394-016-0168-0

Richardson, F. C., \& Suinn, R. M. (1972). The Mathematics Anxiety Rating Scale: Psychometric data. Journal of Counseling Psychology, 19, 551554. http://doi.org/10.1037/h0033456

Schwarz, N. (1999). Self-reports: How the questions shape the answers. American Psychologist, 54, 93-105. https://doi.org/10.1037/0003066X.54.2.93

Shapka, J. D., Domene, J. F., \& Keating, D. P. (2006). Trajectories of career aspirations through adolescence and young adulthood: Early math achievement as a critical filter. Educational Research and Evaluation, 12, 347-358. doi:10.1080/13803610600765752 
Silva, E. (2007). On the clock: Rethinking the way schools use time. Education Sector Reports. Retrieved from http://www.educationsector.org/sites/default/files/publications/Ont heClock.pdf

Sloan, D. M., Marx, B. P., \& Greenberg, E. M. (2011). A test of written emotional disclosure as an intervention for posttraumatic stress disorder. Behaviour Research and Therapy, 49, 299-304. https://doi.org/10.1016/j.brat.2011.02.001

Smyth, J. M., Stone, A. A., Hurewitz, A., \& Kaell, A. (1999). Effects of writing about stressful experiences on symptom reduction in patients with asthma or rheumatoid arthritis. JAMA, 281(14), 1304-1309. https://doi.org/10.1001/jama.281.14.1304

Suárez-Pellicioni, M., Núñez-Peña, M. I., \& Colomé, À. (2015). Math anxiety: A review of its cognitive consequences, psychophysiological correlates, and brain bases. Cognitive, Affective, \& Behavioral Neuroscience. https://doi.org/10.3758/s13415-015-0370-7

Suri, R., Monroe, K. B., \& Koc, U. (2013). Math anxiety and its effects on consumers' preference for price promotion formats. Journal of the Academy of Marketing Science, 41, 271-282. doi:10.1007/s11747-0120313-6

Trice, A., \& Ogden, E. (1986-1987). Correlates of mathematics anxiety in first-year elementary school teachers. Educational Research Quarterly, 11, 2-4.

Vannest, K.J., Parker, R.I., \& Gonen, O. (2011). Single case research: Web based calculators for SCR analysis (Version 1.0) [Web-based application]. College Station, TX: Texas A\&M University. Retrieved from www.singlecaseresearch.org

Young, T. (1980). Teacher time on task: A significant variable. The Phi Delta Kappan, 62, 60. 


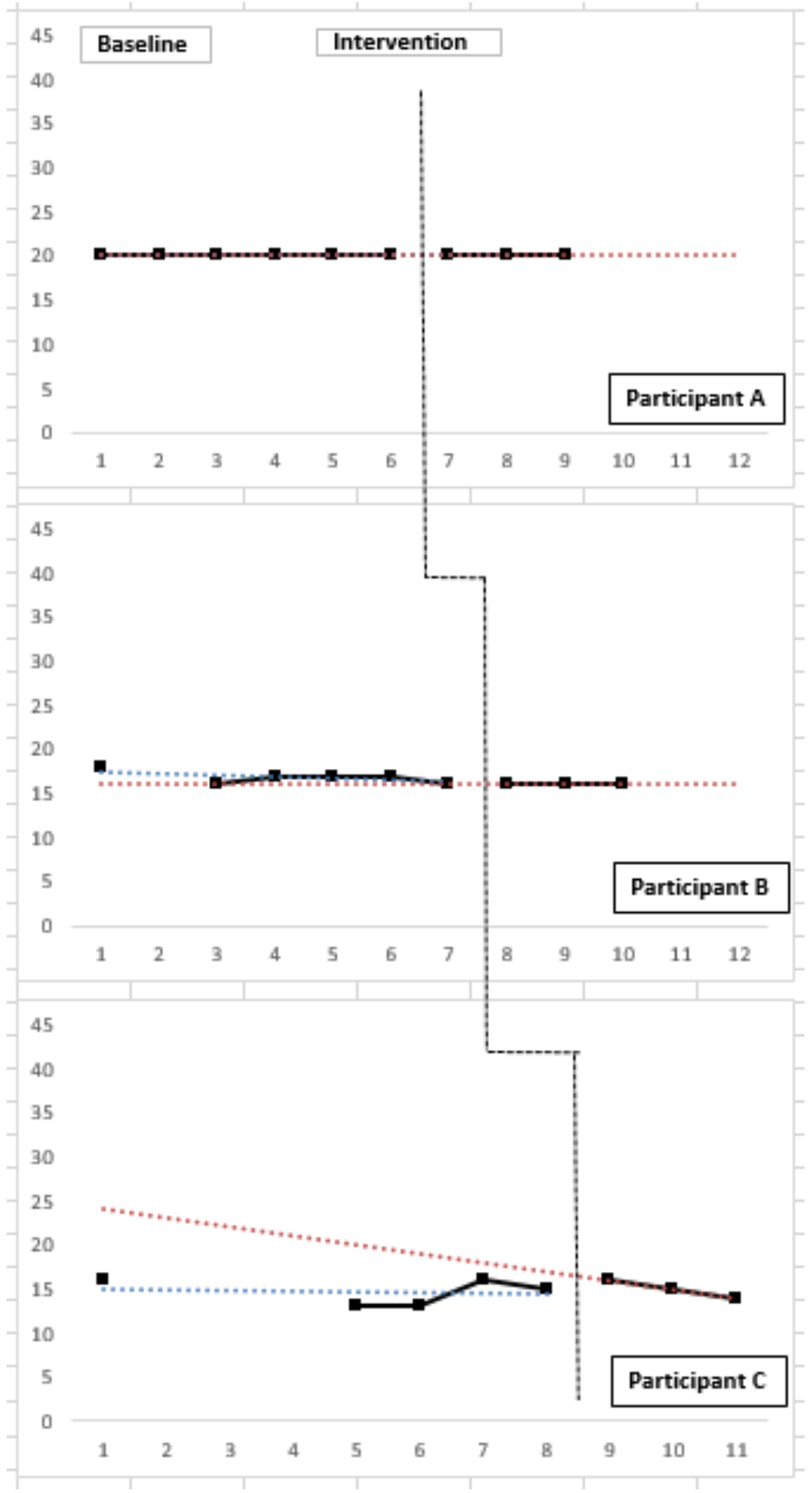

Figure 1. FS-ANX results. 


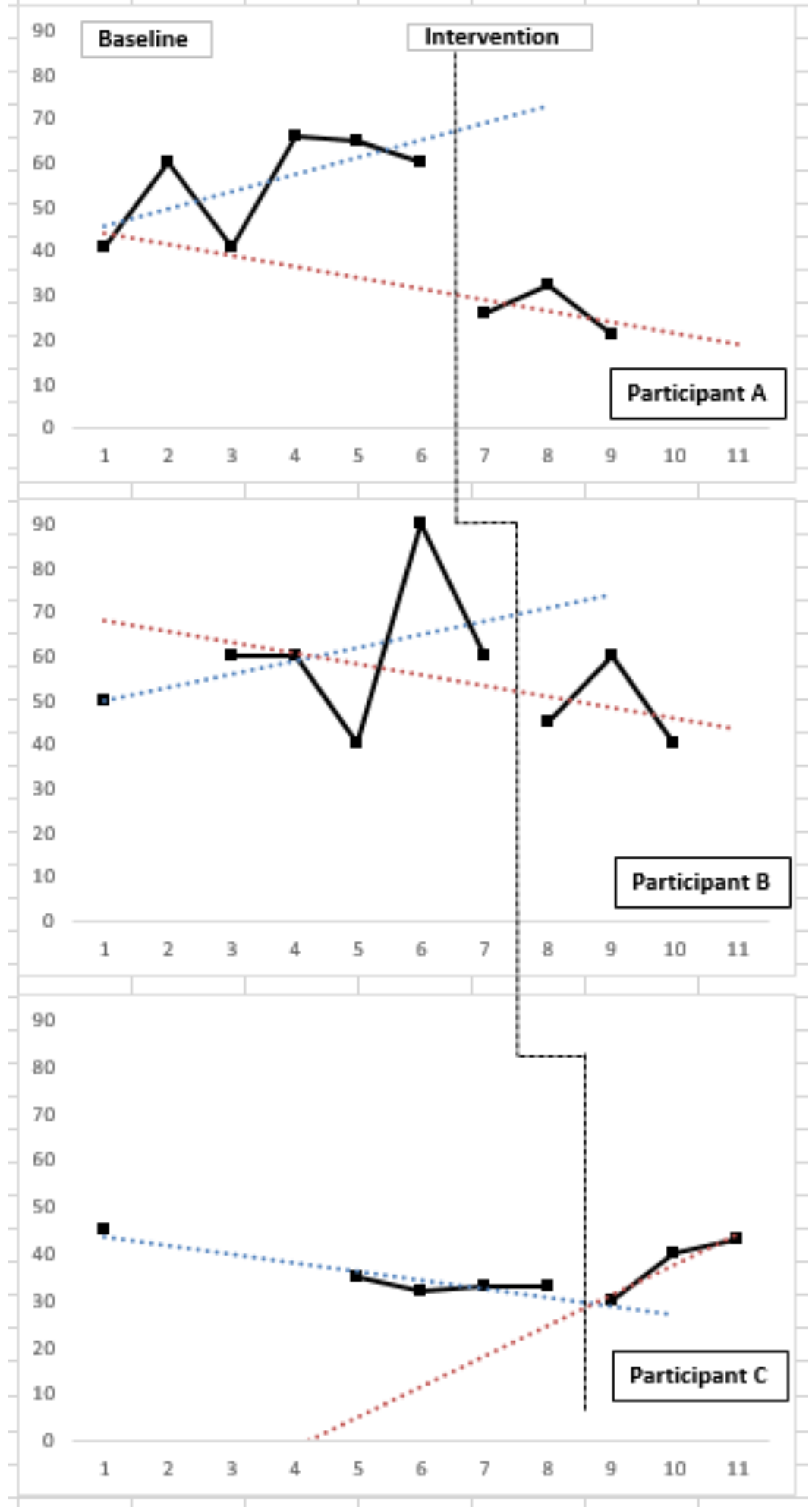

Figure 2. Teacher math-instructional-time results. 